\title{
THE GÖDEL CLASS WITH IDENTITY IS UNSOLVABLE
}

\author{
BY WARREN D. GOLDFARB
}

The Gödel Class with Identity (GCI) is the class of closed, prenex formulas of pure quantification theory extended by inclusion of the identity sign "=" whose prefixes have the form $\forall x \forall y \exists z_{0} \cdots \exists z_{n}$. At the end of [2], Gödel claims that the GCI can be shown to contain no infinity axioms-and hence to be decidable (for satisfiability) - "by the same method" as he employed to show this for the analogous class without identity. (An infinity axiom is a satisfiable formula that has no finite models.) Gödel's claim has been questioned for almost twenty years; since no obvious extension of Gödel's method seemed to apply to the GCI, the decision problem for this class has been deemed open. Gödel's claim is, in fact, erroneous; below we explicitly construct an infinity axiom $F$ in the GCI. Moreover, by exploiting further properties of $F$, we can encode an undecidable problem into the GCI. Hence the GCI is undecidable.

The formula $F$ contains the monadic predicate letter $Z$ and the dyadic letters $S, P_{1}, P_{2}, Q, N, R_{1}, R_{2} . F$ is designed so that, in every model $\mathcal{M}$ of $F$, there will be a unique element $\overline{0}$ such that $\mathcal{M} \vDash Z \overline{0}$, a unique element $\overline{1}$ such that $\mathcal{M} \vDash S \overline{1} \overline{0}$, a unique element $\overline{2}$ such that $\mathcal{M} \vDash S \overline{2} \overline{1}$, and so on ad infinitum. Thus $Z$ acts as the predicate "is zero", and $S$ as the successor relation. The other letters are used to insure the existence of such $\overline{0}, \overline{1}, \overline{2}, \ldots$, and are meant to act as follows. Elements of $\mathcal{M}$ can be taken to encode pairs of integers. Suppose $b$ encodes $\langle p, q\rangle$; then $P_{1}$ holds between $b$ and the element $\bar{p}, P_{2}$ between $b$ and $\bar{q}, Q$ between $b$ and $\overline{q+1}, N$ between $b$ and an element that encodes $\langle p+1, q\rangle, R_{1}$ between $b$ and any element that encodes $\langle q+1, r\rangle$ for some $r$, and $R_{2}$ between $b$ and any element that encodes $\langle r, q+1\rangle$ for some $r$.

Let $F$ be a prenex form of $\forall x \forall y \exists z_{0} H$, where $H$ is the conjunction of the following ten clauses:

(4) $\neg Z x \wedge x \neq y \rightarrow(\exists w)(S x w \wedge-S y w)$;

(5) $\quad S x y \rightarrow(\exists z)\left(Q z x \wedge P_{2} z y \wedge P_{1} z z_{0}\right)$;

Received by the editors August 10, 1983.

1980 Mathematics Subject Classification. Primary 03B10, 03B25; Secondary 68C30.

(C) 1984 American Mathematical Society $0273-0979 / 84 \$ 1.00+\$ .25$ per page 


$$
\begin{aligned}
& (\exists z)\left[N x z \wedge(Q x y \rightarrow Q z y) \wedge\left(R_{1} x y \rightarrow R_{1} z y\right) \wedge\left(R_{2} x y \rightarrow R_{2} z y\right)\right] \\
& N x y \rightarrow(\exists z)\left(P_{2} x z \wedge P_{2} y z\right) \wedge(\exists w)(\exists u)\left(P_{1} x w \wedge S u w \wedge P_{1} y u\right) ; \\
& Q x y \rightarrow(\exists z)\left(P_{1} x z \wedge\left(S y z \rightarrow P_{2} x z\right)\right) ; \\
& \bigwedge_{\delta=1,2}\left[P_{\delta} x y \wedge \neg Z y \rightarrow(\exists z)(\exists w)\left(R_{\delta} z x \wedge P_{2} z w \wedge P_{1} z z_{0} \wedge S y w\right)\right] ; \\
& \bigwedge_{\delta=1,2}\left[R_{\delta} x y \rightarrow(\exists z)(\exists w)\left(P_{1} x z \wedge S w z \wedge\left(P_{\delta} y w \rightarrow P_{2} x z\right)\right)\right] .
\end{aligned}
$$

$F$ is satisfiable. Indeed, let $\pi: \mathbf{N}^{2} \rightarrow \mathbf{N}$ be a bijective pairing function. Interpret the predicate letters over $\mathbf{N}$ as indicated two paragraphs back, where $\overline{0}, \overline{1}, \overline{2}, \ldots$ are identified with $0,1,2, \ldots$ and an integer $k$ is taken to encode $\langle p, q\rangle$ iff $k=\pi(p, q)$. These interpretations yield a model for $F$ with universe $\mathbf{N}$.

Now let $\mathcal{M}$ be any model for $F$. We find distinct elements $\overline{0}, 1,2, \ldots$ of $\mathcal{M}$ such that, for each integer $p$,

(A) for all $c$ in $\mathcal{M}, \neg S \overline{0} c$, and $Z c$ iff $c=\overline{0}$;

(B) for all $c$ in $\mathcal{M}, S \bar{p} c$ iff $p>0$ and $c=\overline{p-1}$;

(C) for all $c$ in $\mathcal{M}$, if $p>0$ and $S c \overline{p-1}$, then $c=\bar{p}$;

(D) for $\delta=1,2$ and all $c, b$ in $\mathcal{M}$, if $P_{\delta} c \bar{p} \wedge P_{\delta} c b$, then $b=\bar{p}$.

(An expression like " $P_{\delta} c b$ " is short for " $\mathcal{M} \vDash P_{\delta} c b$ ".)

By clauses (1) and (2) of $F$, there is a unique $\overline{0}$ in $\mathcal{M}$ such that $Z \overline{0}$. Since the variable $z_{0}$ must always take $\overline{0}$ as its value, clause (2) of $F$ yields (A)-(D) for $p=0$.

As induction hypothesis, suppose $\overline{0}, \ldots, \bar{k}$ are distinct elements of $\mathcal{M}$ obeying (A)-(D) for each $p \leq k$. An $N$-chain is a sequence $\left\langle c_{0}, \ldots, c_{m}\right\rangle$ of elements of $\mathcal{M}$ such that $N c_{i} c_{i+1}$ for each $i<m$. An easy induction on $m$, using clause (7) of $F$ and (C) and (D), yields: for all $p, m \leq k$,

(E) suppose $\left\langle c_{0}, \ldots, c_{m}\right\rangle$ is an $N$-chain; if $P_{2} c_{m} \bar{p}$ then $P_{2} c_{0} \bar{p}$, and if $P_{1} c_{0} \overline{0}$ then $P_{1} c_{m} \bar{m}$.

\section{Lemma 1. Let $a, b \in \mathcal{M}$ and suppose $S a \bar{k}$ and $S a b$. Then $b=\bar{k}$.}

Proof. By clause (5) there exists $c_{0}$ in $\mathcal{M}$ such that $Q c_{0} a \wedge P_{2} c_{0} b \wedge P_{1} c_{0} \overline{0}$. Iterated use of clause (6) yields an $N$-chain $\left\langle c_{0}, \ldots, c_{k}\right\rangle$ such that $Q c_{k} a$. By (E), $P_{1} c_{k} \bar{k}$. By clause (8), there exists $d$ in $\mathcal{M}$ with $P_{1} c_{k} d \wedge\left(S a d \rightarrow P_{2} c_{k} d\right)$. By (D), $d=\bar{k}$; since $S a \bar{k}, P_{2} c_{k} \bar{k}$. By (E), $P_{2} c_{0} \bar{k}$. But $P_{2} c_{0} b$; hence, by (D), $b=\bar{k}$.

\section{Lemma 2. There is a unique $a$ in $\mathcal{M}$ such that $S a \bar{k}$.}

Proof. By clause (3) there is at least one $a$ in $\mathcal{M}$ with $S a \bar{k}$. By (A), $\neg Z a$. Let $b \in \mathcal{M}, b \neq a$. By clause (4) there exists $c$ in $\mathcal{M}$ with $S a c \wedge \neg S b c$. By Lemma $1, c=\bar{k}$. Thus $\neg S b \bar{k}$.

Now let $\overline{k+1}$ be the unique $a$ such that $S a \bar{k}$. By (B), $\overline{k+1}$ is distinct from $\overline{0}, \overline{1}, \ldots, \bar{k}$.

Lemma 3. Let $\delta=1$ or $2, c, b \in \mathcal{M}$; suppose $P_{\delta} c \overline{k+1}$ and $P_{\delta} c b$. Then $b=\overline{k+1}$. 
Proof. By (A) and (D), $\neg Z b$. Hence by clause (9) there exist $c_{0}, d$ in $\mathcal{M}$ such that $R_{\delta} c_{0} c \wedge P_{2} c_{0} d \wedge P_{1} c_{0} 0 \wedge S b d$. Iterated use of clause (6) yields an $N$-chain $\left\langle c_{0}, \ldots, c_{k}\right\rangle$ such that $R_{\delta} c_{k} c$. By (E), $P_{1} c_{k} \bar{k}$. By clause (10) there exist $e, e^{\prime}$ in $\mathcal{M}$ such that $P_{1} c_{k} e \wedge S e^{\prime} e \wedge\left(P_{\delta} c e^{\prime} \rightarrow P_{2} c_{k} e\right)$. By (D), $e=\bar{k}$. Thus $e^{\prime}=\overline{k+1}$. Since $P_{\delta} c \overline{k+1}, P_{2} c_{k} \bar{k}$. By (E), $P_{2} c_{o} \bar{k}$. But $P_{2} c_{0} d ;$ hence, by (D), $d=\bar{k}$. Thus $S b \bar{k}$, so $b=\overline{k+1}$.

Lemmas 1-3 show that (A)-(D) hold for all $p \leq k+1$. Thus, by induction, there is an infinite sequence of distinct elements of $\mathcal{M}$.

We have shown that every model for $F$ contains an $\omega$-sequence of elements on which $S$ acts as the successor relation. Consequently, it is a simple matter to use $F$ to obtain undecidability. For example, let $G=\forall x \exists u \forall y K$ be any $\forall \exists \forall$-formula of pure quantification theory; we may suppose that the predicate letters of $G$ are distinct from those of $F$. A straightforward argument shows that $G$ is satisfiable if and only if $F \wedge \forall x \forall y \exists u(S u x \wedge K)$ is satisfiable; and the latter formula has a prenex equivalent in the GCI. Since the class of $\forall \exists \forall$ formulas is undecidable [3], we obtain the

Theorem. The Gödel Class with Identity is undecidable.

The theorem may be sharpened. Using several additional predicate letters, we may construct an infinity axiom and encode $\forall \exists \forall$-formulas while using only one existential quantifier. Hence the Minimal GCI, i.e., the class of formulas with prefixes $\forall x \forall y \exists z$, is undecidable. This settles the decision problem for all prefix-classes of quantification theory with identity, for we now have the following division:

Decidable prefix-classes: $\exists \cdots \exists \forall \cdots \forall$ and $\exists \cdots \exists \forall \exists \cdots \exists$.

Undecidable prefix-classes: $\forall \exists \forall$ and $\forall \forall \exists$.

This dividing line differs from that in pure quantification theory, where the $\exists . . \exists \forall \forall \exists . . \exists$ class is decidable, so that the minimal undecidable prefix-classes are $\forall \exists \forall$ and $\forall \forall \forall \exists$ (see the Introduction to [1]).

\section{REFERENCES}

1. B. Dreben and W. D. Goldfarb, The decision problem: Solvable classes of quantificational formulas, Addison-Wesley, Reading, Mass., 1979.

2. K. Gödel, Zum Entscheidungsproblem des logischen Funktionenkalküls, Monatsh. Math. Phys. 40 (1933), 433-443.

3. A. S. Kahr, E. F. Moore and H. Wang, Entscheidungsproblem reduced to the $\forall \exists \forall$ case, Proc. Nat. Acad. Sci. U.S.A. 48 (1962), 365-377. 02138

Department of Philosophy, Harvard University, Cambridge, Massachusetts 
